\title{
Vascular Calcification in Patients Undergoing Total Knee Arthroplasty: Frequency and Effects on the Surgery
}

\author{
Ju-Hyung Yoo, MD, Jin-Gyu Kim, MD*, Kwangho Chung, MD*, Seung Hyun Lee, $\mathrm{MD}^{\dagger}$, \\ Hyun-Cheol Oh, MD, Sang-Hoon Park, MD, Sang-Ok Seok, MD \\ Department of Orthopedic Surgery, National Health Insurance Service Ilsan Hospital, Goyang, \\ ${ }^{*}$ Department of Orthopedic Surgery, Yonsei University College of Medicine, Seoul, \\ ${ }^{\dagger}$ Department of Radiology, National Health Insurance Service Ilsan Hospital, Goyang, Korea
}

Background: This study aims to investigate the frequency of distal femoral and popliteal arterial calcification and to evaluate the intraoperative and postoperative effects of arterial calcification in patients undergoing total knee arthroplasty using a tourniquet. Methods: The records of 5,438 patients who had undergone primary total knee arthroplasty between January 2003 and January 2017 were retrospectively reviewed. We examined the preoperative radiographs of the knee from all patients for calcifications of the femoral and popliteal arteries. Vascular calcification was identified on preoperative radiographs in 223 cases. Intraoperative and postoperative complications were investigated among these patients. Postoperative complications were analyzed from the time of surgery to the last follow-up (minimum 1-year follow-up).

Results: Vascular calcification of the arteries around the knee was found in 223 cases (4.1\%). The mean patient age was 70.6 years in the non-calcification group and 73.8 years in the calcification group $(p>0.05)$. The calcification group was classified into medial, intimal, or mixed subgroups according to the morphology of calcification on preoperative radiographs. The medial type included 46 cases (20.6\%); intimal type, 161 cases (72.2\%); and mixed type, 16 cases $(2.7 \%)$. There was no statistically significant difference in demographic and surgical data among the three groups. There were intraoperative complications in two cases in the medial type group, both of which involved tourniquet failure. There was also a postoperative complication in one case in the medial type group, which involved wound dehiscence at 2 weeks postoperatively. No other postoperative complications were identified during 1-year follow-up.

Conclusions: Despite the presence of calcifications in the arteries around the knee, total knee arthroplasty (using a tourniquet) can be performed without serious complications.

Keywords: Knee arthroplasty, Calcification, Tourniquet

Total knee arthroplasty (TKA) is a safe orthopedic surgery, which is performed worldwide in the treatment of patients with advanced knee osteoarthritis. Current studies have

Received August 12, 2019; Accepted September 29, 2019

Correspondence to: Sang-Ok Seok, MD

Department of Orthopedic Surgery, National Health Insurance Service

Ilsan Hospital, 100 Ilsan-ro, Ilsandong-gu, Goyang 10444, Korea

Tel: +82-31-900-3571, Fax: +82-31-900-0343

E-mail: soekso1012@nhimc.or.kr demonstrated satisfactory long-term clinical outcomes and survival rates in patients who underwent TKA. ${ }^{1,2)}$ Despite such excellent results in previous studies, however, the incidence of complications after TKA is as high as $7 \%-12 \%{ }^{3)}$ Common complications after TKA include thromboembolic disease, periprosthetic joint infection, neurovascular injury, periprosthetic fracture, and joint stiffness. ${ }^{4}$

Vascular complications occur very rarely in TKA, but may lead to limb- and life-threatening consequences. ${ }^{5-7)}$ Among the possible vascular complications, isch- 
Yoo et al. Vascular Calcification in Patients Undergoing Total Knee Arthroplasty

Clinics in Orthopedic Surgery • Vol. 12, No. 2, $2020 \bullet$ www.ecios.org

emic complication is the most frequent $(56 \%-60 \%$ of total complications) and serious complication. ${ }^{7-10)}$ There is one case report of acute arterial thrombosis occurring in a patient without the risk factors. ${ }^{11)}$ However, in most cases, ischemic complications develop in patients with vascular calcification; the ischemia results from indirect trauma, which is caused by stretching of the popliteal artery during knee joint dislocation and by blunt pressure applied when using retractors. ${ }^{5}$ Potential ischemic complications include protracted wound healing, wound necrosis, pressureinduced necrosis of the toes, heels, or foot, compartment syndrome, and limb amputation.

Vascular calcifications around the knee are often found on preoperative radiographs in patients who are scheduled to undergo TKA. Lindbom ${ }^{12)}$ classified these vascular calcifications as medial and intimal types based on their morphology on radiographs. It is relatively easy to distinguish between medial and intimal types on plain radiographs. In one study of 39 cadavers by Orr et al., ${ }^{13)}$ the medial/intimal classification based on radiographic criteria correlated with histologic findings in $92 \%$ of cases. Medial artery calcifications are nonobstructive lesions that form a uniform linear "railroad track" on radiographs and increase the stiffness of the arterial wall. ${ }^{14)}$ Intimal calcifications, in contrast, present as discrete plaque-like dense clumps (Fig. 1). ${ }^{10,14)}$

Pneumatic tourniquets are often used in TKA to improve structure visualization, reduce intraoperative
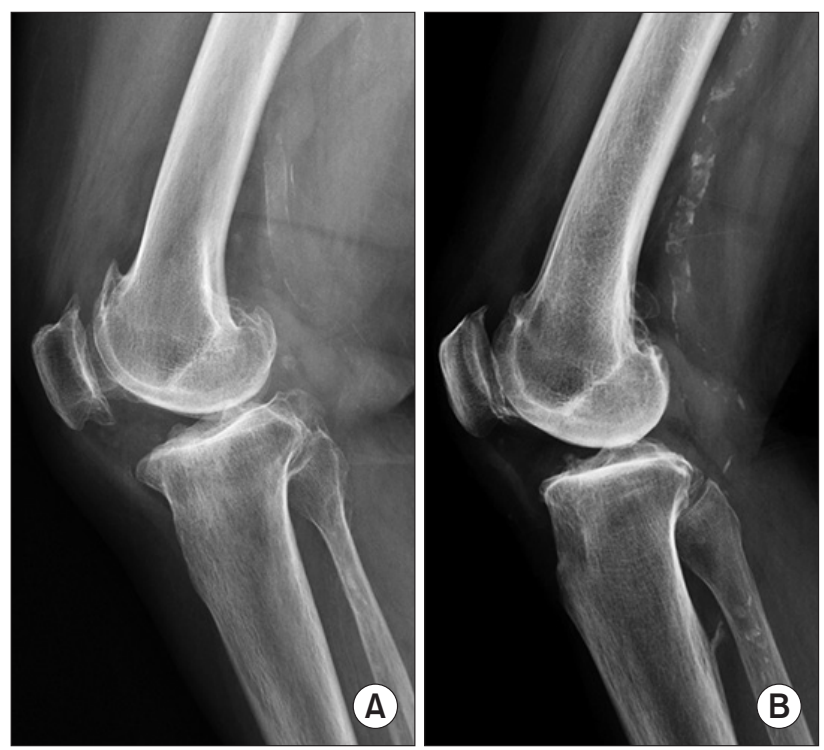

Fig. 1. Preoperative lateral radiographs of the knee. (A) Medial artery calcifications are observed as a uniform linear railroad track. (B) Intimal calcifications present as discrete plaque-like dense clumps. bleeding, and obtain a better cement-bone interface intraoperatively. ${ }^{15)}$ However, tourniquets also have risk of adverse effects. They have been associated with nerve palsies, vascular injuries, postoperative pain, and muscle damage. ${ }^{15,16)}$ There is particular concern for ischemic complications in cases of TKA performed using a tourniquet when there are calcifications in the femoral and/or popliteal arteries on preoperative radiographs. ${ }^{8,17)}$ In addition, some studies have found that the presence of arterial calcifications around the knee on preoperative radiographs was associated a higher risk of postoperative cardiovascular events and mortality. ${ }^{18,19)}$ The purpose of this study was to evaluate the incidence and characteristics of calcifications around the knee joint and the incidence of intraoperative and postoperative complications in patients who underwent TKA using a tourniquet.

\section{METHODS}

We conducted this study in compliance with the principles of the Declaration of Helsinki. The protocol of this study was reviewed and approved by the Institutional Review Board of National Health Insurance Service Ilsan Hospital (IRB No. 2019-04-002). Written informed consents were obtained. The records of 5,438 patients who underwent primary TKA at our institution between January 2003 and January 2017 were retrospectively reviewed. The indications for surgery included degenerative arthritis in 5,406 cases, rheumatoid arthritis in 18 cases, osteonecrosis in 10 cases, traumatic arthritis in three cases and gouty arthritis in one case. There were 358 men, and 5,080 women included. The left knee was operated on in 2,735 cases and the right knee, in 2,667 cases.

We examined the preoperative knee radiographs from all patients for femoral and/or popliteal artery calcifications. Calcifications were classified as medial, intimal, or mixed. ${ }^{12,14)}$ Radiologic evaluation was performed by an orthopedic surgeon (JHY) and a radiologist (SHL). The orthopedic surgeon first judged the presence of calcification of vessels in the preoperative radiographs. The calcifications were later confirmed and classified by the radiologist.

Vascular calcifications were identified on the preoperative knee radiographs of 223 patients. We reviewed patient age, body mass index (BMI), sex, medical comorbidities, and surgical time. Intraoperative and postoperative complications were investigated among these patients. Postoperative complications were analyzed from the time of surgery to the date of last follow-up (minimum 1 year, range, 1 year to 12 years 7 months). Systemic complica- 
Yoo et al. Vascular Calcification in Patients Undergoing Total Knee Arthroplasty

Clinics in Orthopedic Surgery • Vol. 12, No. 2, $2020 \bullet$ www.ecios.org

tions and ischemic complications were also investigated.

One senior surgeon (JHY) performed all of the operations. A cemented TKA with patellar resurfacing was used in all patients. The LCS Total Knee System (DePuySynthes, Warsaw, IN, USA) implant was used in two cases, while the NexGen LPS-Flex Total Knee System (Zimmer, Warsaw, IN, USA) was used in the remaining 221 cases. The surgery was performed by using the conventional parapatellar approach in five cases and the minimally invasive approach in 218 cases. The indications for surgery in these patients included degenerative arthritis in 222 cases and osteonecrosis in one case. A tourniquet with an inherent pressure of $320 \mathrm{mmHg}$ was used in all patients during TKA. The tourniquet was inflated immediately before skin incision and was deflated after wound closure. A closed suction drain was inserted in the knee joint and was removed 48 hours after surgery in all patients.

Deep vein thrombosis prophylaxis was performed by using a foot pump for 72 hours after surgery and compression stockings were worn for 6 weeks after surgery in all patients. A blood clot buster was not used. A passive knee joint exercise using a passive motion machine for joints and walker-aided walking were started on the 2nd postoperative day, depending on the condition of the patient.

Pearson chi-square test was used to analyze nominal variables. The $t$-test, Mann-Whitney test, and oneway analysis of variance were used to compare continuous variables and to determine significant differences. All statistical analyses were performed by using IBM SPSS ver. 25.0 (IBM Corp., Armonk, NY, USA). The statistical significance level was set at $p<0.05$ for all tests.

\section{RESULTS}

Vascular calcifications of the artery around the knee were identified in 223 cases (4.1\%) in total, including 105 cases (3.84\%) on the left side and 118 cases $(4.42 \%)$ on the right side $(p>0.05)$. The incidence of calcifications in men was $7.0 \%$ (25 of 358 cases) and 3.9\% (198 of 5,080 cases) in women $(p>0.05)$. The mean ( \pm standard deviation) patient age was $70.6( \pm 6.0)$ years in the non-calcification group and $73.8( \pm 5.7)$ years in the calcification group $(p>$ 0.05) (Table 1).

The medial type included 46 cases (20.6\%); intimal type, 161 cases (72.2\%); and mixed type, 16 cases (2.7\%). The mean ( \pm standard deviation) patient age was $73.07( \pm 6.0)$ years in the medial type, $74.23( \pm 5.7)$ in the intimal type, and $72.25( \pm 4.8)$ in the mixed type $(p>0.05)$. The mean BMI was $26.72 \mathrm{~kg} / \mathrm{cm}^{2}$ in the medial type, $25.56 \mathrm{~kg} / \mathrm{cm}^{2}$ in the intimal type, and $26.52 \mathrm{~kg} / \mathrm{cm}^{2}$ in the mixed type ( $p>$ 0.05 ). The total blood loss was $825 \pm 288 \mathrm{~mL}$ in the medial type, $760 \pm 400$ in the intimal type, and $745 \pm 333$ in the mixed type $(p>0.05)$. There were no statistically significant differences in demographic or surgical data between the three groups (Tables 2 and 3 ).

Medical comorbidities were also evaluated in the calcification group. Hypertension was the most common comorbidity, which was observed in 203 patients (91.0\%). There were 114 cases (51.5\%) of diabetes mellitus, 14 cases (6.3\%) of cerebrovascular disease, and eight cases (3.6\%) of coronary artery disease $(p>0.05)$. Similarly, there were no statistically significant differences in the frequency of medical comorbidities in the three groups (Table 4).

Table 1. Demographic Data

\begin{tabular}{cccc} 
Variable & $\begin{array}{c}\text { Patient with } \\
\text { calcification } \\
\text { on preoperative } \\
\text { radiograph } \\
(n=223)\end{array}$ & $\begin{array}{c}\text { Patient without } \\
\text { calcification } \\
\text { on preoperative } \\
\text { radiograph } \\
(n=5,215)\end{array}$ & $p$-value \\
\hline Age (yr), mean \pm SD & $73.8 \pm 5.7$ & $70.6 \pm 6.0$ & NS \\
Sex (male : female) & $25: 198$ & $333: 4,882$ & NS \\
\hline
\end{tabular}

SD: standard deviation, NS: not significant.

Table 2. Demographic Data of Patients with Calcifications on Preoperative Radiographs

\begin{tabular}{|c|c|c|c|c|}
\hline Variable & $\begin{array}{l}\text { Medial type } \\
\qquad(n=46)\end{array}$ & $\begin{array}{l}\text { Intimal type } \\
(\mathrm{n}=161)\end{array}$ & $\begin{array}{l}\text { Mixed type } \\
\quad(n=16)\end{array}$ & $p$-value \\
\hline Age (yr), mean \pm SD & $73.07 \pm 6.0$ & $74.23 \pm 5.7$ & $72.25 \pm 4.8$ & NS \\
\hline Sex (male : female) & $8: 38$ & $15: 146$ & $2: 14$ & NS \\
\hline Right : left & $26: 20$ & $84: 77$ & $9: 7$ & NS \\
\hline $\begin{array}{c}\mathrm{BMI}\left(\mathrm{kg} / \mathrm{cm}^{2}\right) \\
\text { mean } \pm \mathrm{SD}\end{array}$ & $26.72 \pm 4.3$ & $25.56 \pm 3.5$ & $26.52 \pm 3.9$ & NS \\
\hline
\end{tabular}

SD: standard deviation, NS: not significant, BMI: body mass index.

Table 3. Surgical Data of Patients with Calcifications on Preoperative Radiographs

\begin{tabular}{ccccc}
\multicolumn{1}{c}{ Variable } & $\begin{array}{c}\text { Medial type } \\
(\mathrm{n}=46)\end{array}$ & $\begin{array}{c}\text { Intimal type } \\
(\mathrm{n}=161)\end{array}$ & $\begin{array}{c}\text { Mixed type } \\
(\mathrm{n}=16)\end{array}$ & $p$-value \\
\hline Operation time $(\mathrm{min})$ & $83.1 \pm 20.5$ & $77.3 \pm 12.9$ & $77.3 \pm 16.3$ & NS \\
\hline Total blood loss $(\mathrm{mL})$ & $825 \pm 288$ & $760 \pm 400$ & $745 \pm 333$ & NS \\
\hline
\end{tabular}

Values are presented as mean \pm standard deviation.

NS: not significant. 
Yoo et al. Vascular Calcification in Patients Undergoing Total Knee Arthroplasty

Clinics in Orthopedic Surgery • Vol. 12, No. 2, $2020 \bullet$ www.ecios.org

\begin{tabular}{|c|c|c|c|c|}
\hline Comorbidity & $\begin{array}{l}\text { Medial type } \\
(n=46)\end{array}$ & $\begin{array}{l}\text { Intimal type } \\
(n=161)\end{array}$ & $\begin{array}{l}\text { Mixed type } \\
(n=16)\end{array}$ & $p$-value \\
\hline Hypertension & 38 (82.60) & 151 (93.20) & $15(93.80)$ & NS \\
\hline Diabetes mellitus & $26(56.20)$ & 79 (48.50) & $25(62.50)$ & NS \\
\hline $\begin{array}{l}\text { Coronary occlusive } \\
\text { disease }\end{array}$ & $27(57.10)$ & $34(21.10)$ & - & NS \\
\hline $\begin{array}{l}\text { Cerebrovascular } \\
\text { disease }\end{array}$ & $2(4.50)$ & $12(7.60)$ & - & NS \\
\hline
\end{tabular}

Values are presented as number (\%).

NS: not significant.

Ischemic complications were found in three cases $(0.06 \%$, three of 5,215 cases) in the non-calcification group and in three cases (1.35\%, three of 223 cases) in the calcification group. The incidence rate of ischemic complications was significantly higher in patients with preoperative vascular calcification than in patients without preoperative vascular calcification $(p<0.05)$. All three cases in the non-calcification group were postoperative complications: wound dehiscence in two cases and wound necrosis in one case. There were two intraoperative complications and one postoperative complication in the calcification group. Intraoperative complications were noted in two cases in the medial type. All intraoperative complications involved tourniquet failure. Despite the increase in the tourniquet pressure to $370 \mathrm{mmHg}$ intraoperatively in these cases, the tourniquet did not work effectively, and intraoperative bleeding continued. There was one postoperative complication in the medial-type group, which involved wound dehiscence after 2 postoperative weeks. No other postoperative complications were identified after at least 1 year of follow-up (range, 1 year to 12 years 7 months).

\section{DISCUSSION}

In this study, the incidence of vascular calcifications on preoperative knee radiographs in patients who underwent TKA was $4.1 \%$. Patients with preoperative calcifications tended to be older than were those without calcifications, although this difference was not statistically significant. The intimal type of calcification was the most common type (72.2\%), followed by medial (20.6\%) and mixed types (2.7\%). Regarding medical comorbidities, diabetes mellitus and coronary occlusive disease tended to be more common in the medial type, while hypertension and cerebrovascular disease were more common in the intimal type. However, the difference was not statistically significant. Intraoperative and postoperative complications were identified in all three cases, and all were observed in the medial type.

Although vascular complications after TKA are rare, they can have devastating consequences, such as pulmonary embolism, compartment syndrome, amputation, or even mortality. ${ }^{502-22)}$ The risk of ischemic complications may be increased in patients with a history of peripheral vascular disease. Arterial calcifications can decrease an artery's elasticity and compliance. Therefore, these arteries may be more vulnerable to rapid occlusion or rupture in response to external stimuli than are arteries without calcification. In their study of 4,097 patients who underwent TKA, Calligaro et al. ${ }^{23}$ reported that arterial thrombosis and ischemia only occurred in patients with preexisting peripheral vascular disease. Similarly, in a study of 1,182 patients, Smith et al. ${ }^{24)}$ concluded that the risk factors for arterial complication after TKA included a history of arterial insufficiency and radiologic evidence of calcifications around the knee. If any of these factors is present on the preoperative assessment, the TKA may have to be performed without a tourniquet.

We identified calcifications on plain radiographs in 223 of 5,438 cases (4.1\%). This prevalence of calcifications was lower than that found in previous studies. WoelfleRoos et al. ${ }^{10)}$ reported that patients with calcifications around the knee joints made up approximately $32 \%$ of all patients scheduled for TKA. Among these patients with calcification, the medial type was found in $20.1 \%$ and the intimal type in $79.9 \%$. Cantu Morales et al. ${ }^{19)}$ observed calcifications in $21.1 \%$ of 900 patients who underwent TKA.

Medial arterial calcification, or Mönckeberg's arteriosclerosis, is a condition that leads to stiffening of the elastic layer of the arterial wall. It does not cause narrowing of the arterial lumen and is usually asymptomatic. ${ }^{25)}$ However, medial calcification is related to aging and diabetes, can reduce artery elasticity, and is a strong predictor of cardiovascular mortality. ${ }^{14)}$ In our study, the incidence of diabetes mellitus in patients with medial type calcification was $56.5 \%$, which was higher than in those with intimal type calcification (48.5\%). However, there was no significant difference.

Intimal calcifications represent the presence of intravascular plaques and may lead to diminished organ perfusion. ${ }^{13,14,25)}$ Woelfle-Roos et al. ${ }^{10)}$ retrospectively evaluated 825 patients who underwent TKA using a tourniquet and concluded that patients with intimal artery calcifications had a higher risk of ischemic complications, such as protracted wound healing, than those without these calcifica- 
tions. Nevertheless, most of these patients had a normal distal pulsation, no clinical signs of claudication, and no history of peripheral artery disease. Furthermore, preoperative X-rays of patients with intimal calcifications may be easily overlooked, because only one or two small plaques are observed. Therefore, one must have a high index of suspicion to detect calcifications on preoperative radiographs, especially in cases of intimal artery calcifications.

Several previous studies did not recommend the use of tourniquets in patients with vascular calcifications who underwent TKA. These studies suggested that the use of a tourniquet increases the risk of vascular complications. ${ }^{17,23,24)}$ The mechanism to explain this hypothesis is that mechanical pressure from the tourniquet causes plaque dislodgement in the blood vessel and eventual occlusion of distal blood flow. ${ }^{26)}$ There are also possible complications associated with the use of tourniquets on calcified arteries, including rupture of the arterial wall, aneurysm formation, and continuous intraoperative bleeding due to ineffective arterial occlusion. ${ }^{4)}$

Kobayashi et al. ${ }^{27)}$ reported that the cause of arterial occlusion was thrombosis initiated by a plaque disruption in the popliteal artery during manipulation of the knee. They recommended that in patients with vascular calcifications around the knee or in the abdomen, TKA should be performed without a tourniquet. They also specified that intraoperative knee joint and soft-tissue manipulation should be done carefully.

Koehler et al ${ }^{15)}$ retrospectively reviewed $373 \mathrm{pa}-$ tients who underwent TKA using a tourniquet. Arterial calcifications did not increase the risk of complications. They concluded that tourniquets can be used safely in patients with arterial calcifications who undergo TKA. Vivacqua et $\mathrm{al}^{4)}$ found that there were no differences in the rates of postoperative complications between a group of 58 patients with arterial calcifications and a control group without calcification. They demonstrated that performing TKA with the help of a tourniquet is a useful method that does not increase the surgical risk. Our result was consistent with these studies. In 223 patients with calcifications on preoperative radiographs, TKA was performed using a tourniquet. Only one postoperative ischemic complication occurred in a patient with medial type calcification, which involved delayed wound healing. It is not clear whether the cause of the wound problem was related to the calcifi- cation. No other postoperative systemic complications or local complications were found.

Hozack et al. ${ }^{28)}$ reported that the vascular complications after TKA are preventable. Popliteal calcifications on plain radiographs and family history of arteriosclerotic disease were possible risk factors for vascular complications. Therefore, careful preoperative evaluations, including review of past medical history, family history, and peripheral vascular physical examination, are necessary.

There were two intraoperative complications in which bleeding was sustained during surgery because the tourniquet failed to occlude the artery. In two cases of medial type calcification, there was continuous bleeding even after an increase in pressure on the tourniquet from 320 to $370 \mathrm{mmHg}$. Increased stiffness of a calcified artery can lead to tourniquet failure. In this case, a large amount of continuous bleeding may occur during the surgery, because the tourniquet compresses the vein without compressing the artery. ${ }^{16}$ Several case reports have described calcified vessels that were resistant to collapse with tourniquet pressures of $300 \mathrm{mmHg}, 450 \mathrm{mmHg}, 500 \mathrm{mmHg}$, or even $600 \mathrm{mg}$ in TKA. ${ }^{29)}$ Tourniquet pressure can be continuously increased until the calcified arteries are compressed; however, this excessive pressure can cause nerve damage, delayed wound healing, surgical site infection, and even popliteal artery rupture. ${ }^{30)}$ When a tourniquet does not effectively occlude the artery in a patient with calcifications, TKA should be performed without a tourniquet to reduce intraoperative blood loss.

The main weakness of this study is its retrospective design. Therefore, we could not completely exclude the bias in our radiographic evaluations. To overcome this limitation, the orthopedic surgeon and radiologist involved were blinded to the clinical data. And there was no radiologic evaluation performed postoperatively to assess the change of the vascular condition compared with preoperative radiographs. The main strength of this study is that we investigated a large study group. In conclusion, despite calcifications in the arteries around the knee, TKA using a tourniquet did not cause serious complications.

\section{CONFLICT OF INTEREST}

No potential conflict of interest relevant to this article was reported. 
Yoo et al. Vascular Calcification in Patients Undergoing Total Knee Arthroplasty

Clinics in Orthopedic Surgery • Vol. 12, No. 2, $2020 \bullet$ www.ecios.org

\section{REFERENCES}

1. Ritter MA. The Anatomical Graduated Component total knee replacement: a long-term evaluation with 20-year survival analysis. J Bone Joint Surg Br. 2009;91(6):745-9.

2. Georgaklis VA, Karachalios T, Makridis KG, et al. Genesis 1 posterior cruciate-retaining total knee arthroplasty with asymmetric tibial tray: an 18-to-26-year long-term clinical outcome study. Knee. 2019;26(4):838-46.

3. Morrey BF, Adams RA, Ilstrup DM, Bryan RS. Complications and mortality associated with bilateral or unilateral total knee arthroplasty. J Bone Joint Surg Am. 1987;69(4):4848.

4. Vivacqua T, Barroso M, Matos P, Pires E Albuquerque R, Cavanellas N, Barretto JM. The use of tourniquet during total knee replacement in patients with and without popliteal artery calcification. Rev Bras Ortop. 2018;53(2):165-70.

5. Parvizi J, Pulido L, Slenker N, Macgibeny M, Purtill JJ, Rothman RH. Vascular injuries after total joint arthroplasty. J Arthroplasty. 2008;23(8):1115-21.

6. Abularrage CJ, Weiswasser JM, Dezee KJ, Slidell MB, Henderson WG, Sidawy AN. Predictors of lower extremity arterial injury after total knee or total hip arthroplasty. J Vasc Surg. 2008;47(4):803-8.

7. Calligaro KD, Dougherty MJ, Ryan S, Booth RE. Acute arterial complications associated with total hip and knee arthroplasty. J Vasc Surg. 2003;38(6):1170-7.

8. DeLaurentis DA, Levitsky KA, Booth RE, et al. Arterial and ischemic aspects of total knee arthroplasty. Am J Surg. 1992;164(3):237-40.

9. Wilson JS, Miranda A, Johnson BL, Shames ML, Back MR, Bandyk DF. Vascular injuries associated with elective orthopedic procedures. Ann Vasc Surg. 2003;17(6):641-4.

10. Woelfle-Roos JV, Dautel L, Wernerus D, Woelfle KD, Reichel $H$. Vascular calcifications on the preoperative radiograph: predictor of ischemic complications in total knee arthroplasty? J Arthroplasty. 2016;31(5):1078-82.

11. Bayne CO, Bayne O, Peterson M, Cain E. Acute arterial thrombosis after bilateral total knee arthroplasty. J Arthroplasty. 2008;23(8):1239.

12. Lindbom A. Arteriosclerosis and arterial thrombosis in the lower limb: a roentgenological study. Acta Radiol Suppl. 1950;80:1-80.

13. Orr DP, Myerowitz RL, Herbert DL, Friday P. Correlation of radiographic and histologic findings in arterial calcification. Invest Radiol. 1978;13(2):110-4.

14. Lehto S, Niskanen L, Suhonen M, Ronnemaa T, Laakso M. Medial artery calcification: a neglected harbinger of cardio- vascular complications in non-insulin-dependent diabetes mellitus. Arterioscler Thromb Vasc Biol. 1996;16(8):978-83.

15. Koehler SM, Fields A, Noori N, Weiser M, Moucha CS, Bronson MJ. Safety of tourniquet use in total knee arthroplasty in patients with radiographic evidence of vascular calcifications. Am J Orthop (Belle Mead NJ). 2015;44(9):E308-16.

16. Barr L, Iyer US, Sardesai A, Chitnavis J. Tourniquet failure during total knee replacement due to arterial calcification: case report and review of the literature. J Perioper Pract. 2010;20(2):55-8.

17. Abu Dakka M, Badri H, Al-Khaffaf H. Total knee arthroplasty in patients with peripheral vascular disease. Surgeon. 2009;7(6):362-5.

18. Rennenberg RJ, Kessels AG, Schurgers LJ, van Engelshoven JM, de Leeuw PW, Kroon AA. Vascular calcifications as a marker of increased cardiovascular risk: a meta-analysis. Vasc Health Risk Manag. 2009;5(1):185-97.

19. Cantu Morales D, de Beer J, Petruccelli D, Kabali C, Winemaker M. Lower extremity arterial calcification on preoperative knee radiographs as a predictor of postoperative cardiovascular events after primary total knee arthroplasty. J Arthroplasty. 2018;33(4):1181-5.

20. Bernhoff $K$, Bjorck M. Iatrogenic popliteal artery injury in non arthroplasty knee surgery. Bone Joint J. 2015;97(2):192-6.

21. Bernhoff K, Rudstrom H, Gedeborg R, Bjorck M. Popliteal artery injury during knee replacement: a population-based nationwide study. Bone Joint J. 2013;95(12):1645-9.

22. Da Silva MS, Sobel M; Surgeons of the Southern Association of Vascular Surgery. Popliteal vascular injury during total knee arthroplasty. J Surg Res. 2003;109(2):170-4.

23. Calligaro KD, DeLaurentis DA, Booth RE, Rothman RH, Savarese RP, Dougherty MJ. Acute arterial thrombosis associated with total knee arthroplasty. J Vasc Surg. 1994;20(6):92732.

24. Smith DE, McGraw RW, Taylor DC, Masri BA. Arterial complications and total knee arthroplasty. J Am Acad Orthop Surg. 2001;9(4):253-7.

25. Sucker C, Lanzer P. Arteriosclerosis and media sclerosis: a comparison of 2 calcifying vascular diseases. Med Klin (Munich). 2000;95(4):207-10.

26. Parfenchuck TA, Young TR. Intraoperative arterial occlusion in total joint arthroplasty. J Arthroplasty. 1994;9(2):21720.

27. Kobayashi S, Isobe K, Koike T, Saitoh S, Takaoka K. Acute arterial occlusion associated with total knee arthroplasty. Arch Orthop Trauma Surg. 1999;119(3-4):223-4. 
Yoo et al. Vascular Calcification in Patients Undergoing Total Knee Arthroplasty

Clinics in Orthopedic Surgery • Vol. 12, No. 2, $2020 \bullet$ www.ecios.org

28. Hozack WJ, Cole PA, Gardner R, Corces A. Popliteal aneurysm after total knee arthroplasty: case reports and review of the literature. J Arthroplasty. 1990;5(4):301-5.

29. Woelfle-Roos JV, Dautel L, Mayer B, Bieger R, Woelfle $\mathrm{KD}$, Reichel H. Vascular calcifications on the preoperative radiograph: harbinger of tourniquet failure in patients undergoing total knee arthroplasty? Skeletal Radiol.
2017;46(9):1219-24.

30. Olivecrona C, Ponzer S, Hamberg P, Blomfeldt R. Lower tourniquet cuff pressure reduces postoperative wound complications after total knee arthroplasty: a randomized controlled study of 164 patients. J Bone Joint Surg Am. 2012;94(24):2216-21. 